\title{
Broad-band parallel studies of Cygnus X-1 and GRS 1915+105
}

\author{
Marion Cadolle Bel*, Björn Lehnert \\ ESAC/ESA, Villañueva de la Cañada, Madrid, Spain \\ E-mail: Marion. Cadolle@sciops.esa.int
}

\section{Jérôme Rodriguez}

CEA-Saclay, AIM, France

\section{Diana Hannikainen}

Metsahovi Radio Observatory, Helsinki University of Technology, Finland

\section{Simon Shaw}

School of Physics and Astronomy, University of Southampton, UK

\section{Julien Malzac}

CESR Toulouse, France

\section{Michel Tagger}

CEA-Saclay, APC, CNRS Orleans, France

\section{Peggy Varnière}

CNRS, APC Paris, France

We present INTEGRAL, RXTE, MAGIC and radio data together with spectro-temporal analysis of these two famous BH binaries. Cygnus X-1 was seen by INTEGRAL during a bright event that occurred in 2006 September simultaneously with a detection at $0.1-1 \mathrm{TeV}$ energies by the MAGIC telescope. The source was in a remarkably stable Low/Hard State over a few days, with however a flux as high as $2 \mathrm{Crab}$ ( $\sim 3 \%$ of the Eddington luminosity). A similar hard flare lasting about 8 hours reached the maximum of $2 \mathrm{Crab}$ in the $40-80 \mathrm{keV}$ band on 2008 May 18. We compare the constraints derived in both cases on the accretion flow. In parallel, we summarize the results of a monitoring RXTE, INTEGRAL and Ryle campaign on GRS 1915+105: several discrete ejections occurred (seen for the 1 st time in the lambda class) as a response to a hard X-ray dip and then a disappearance of the $>18 \mathrm{keV}$ emission. We identify the trigger of the ejection as an X-ray spike (energy and material accumulated then ejected) in several variability classes while a possible relation is found between the amplitude of the radio flare and the duration of the dip.

VII Microquasar Workshop: Microquasars and Beyond

September 1-5 2008

Foca, Izmir, Turkey

\footnotetext{
* Speaker.
} 


\section{Introduction}

Cygnus X-1 is the prototype of black hole binaries. Since its discovery in 1964 (Bowyer et al. 1965), it has been intensively observed by all the high energy instruments, from soft Xrays to $\gamma$-rays. It is a persistent source, powered by accretion onto a black hole from a massive companion, HDE 226868, most likely via a focused wind. The value of the mass of the black hole is subject to controversy, it is in the range of (5-15) $M_{\odot}$ according to Herrero et al. (1995) or (14-27) $M_{\odot}$ (Ziólkowski 2005; see also Gies \& Bolton 1986). The distance, $D$, is most likely within $D \sim 2.1 \pm 0.2 \mathrm{kpc}$ (Ziólkowski 2005 and references therein); hereafter we adopt $D=2 \mathrm{kpc}$. This source is most often observed in the so-called low/hard state but occasionally switches to the high/soft state. In recent years, Cygnus X-1 also often appeared in intermediate states in which the source exhibits spectra that are intermediate between the two canonical spectral states and with a strong flaring activity (e.g., Cadolle Bel et al. 2006, Malzac et al. 2006).

GRS 1915+105 has been extensively observed with RXTE since 1996. A rich pattern of variability has emerged from these data with time scales from years down to $15 \mathrm{~ms}$ (e.g. Morgan, Remillard \& Greiner 1997). Belloni et al. (2000), analyzing 163 RXTE observations from 199697 , classified all the observations into 12 separate classes (labeled with greek letters) based on count rates and color characteristics. This scheme has been widely used ever since and is also applied here. The classes could be interpreted as transitions between three basic states (A-B-C): A being equivalent to the Soft State, B to the Soft Intermediate State and C to the Hard Intermediate state in the classification of Homan \& Belloni (2005). These spectral changes are, in most of the classes, interpreted as reflecting the rapid disappearance of the inner portions of an accretion disc, followed by a slower refilling of the emptied region (Belloni et al. 1997). Note that other possibilities as the disappearance of the corona (Rodriguez et al. 2002; Vadawale et al. 2003), or dissipation of magnetic energy (e.g. Tagger et al. 2004) have also been invoked in some models.

\section{Observations and Data Reduction}

We analyse the hard X-ray emission (light curves and spectra) from 18 to $700 \mathrm{keV}$ with the INTEGRAL data taken on 2006 September 24-26 by the IBIS and SPI instruments and which was simultaneous with a detection at $0.15-1 \mathrm{TeV}$ energies by the MAGIC telescope (Albert et al. 2007). These data are supplemented with RXTE All Sky Monitor data at lower energy. Using the standard procedures of data analysis, we present the light curves and fit the high-energy spectrum. We also compare this 2006 event with the another more one which occurred on 2008 May 18.

Besides, we summarize in this work a large multiwavelength campaign of INTEGRAL, RXTE and Ryle data taken from 2004 October to 2005 December. For more details on data log and reduction, we refer the reader to Rodriguez et al. (2008a, b). We report, in a model independent way, the multi-wavelength results from our 2 years monitoring campaign, focusing on the observations during which correlated X-ray and radio variabilities are seen and studying spectral/temporal properties. 


\section{Results}

\subsection{Cygnus X-1}

During the two-day observation, the flux of the source varied significantly and reached values $>2$ Crab (Fig.1, left), among the highest daily fluxes observed by CGRO/BATSE from 1991 to 2000 in the $100-300 \mathrm{keV}$ band and exceeded all of them in the $20-100 \mathrm{keV}$ band (Zdziarski et al. 2002). This event is also particularly interesting with regards to the simultaneous, and the first ever, detection of Cygnus X-1 at TeV energies by the MAGIC telescope on 2006 September 24: the 0.1-1 TeV luminosity during the MAGIC observation was $\sim 2.2 \times 10^{34} \mathrm{erg} \mathrm{s}-1$, whereas the X-ray flare luminosity was about $5 \times 10^{37} \mathrm{erg} \mathrm{s}^{-1}$. Further INTEGRAL observations of Cygnus X-1 taken on 2006 September 30 indicated that the source returned to a more usual state at a level of $\sim 1 \mathrm{Crab}$ in the $20-40 \mathrm{keV}$ band and $1.3 \mathrm{Crab}$ in the $40-80 \mathrm{keV}$ band.
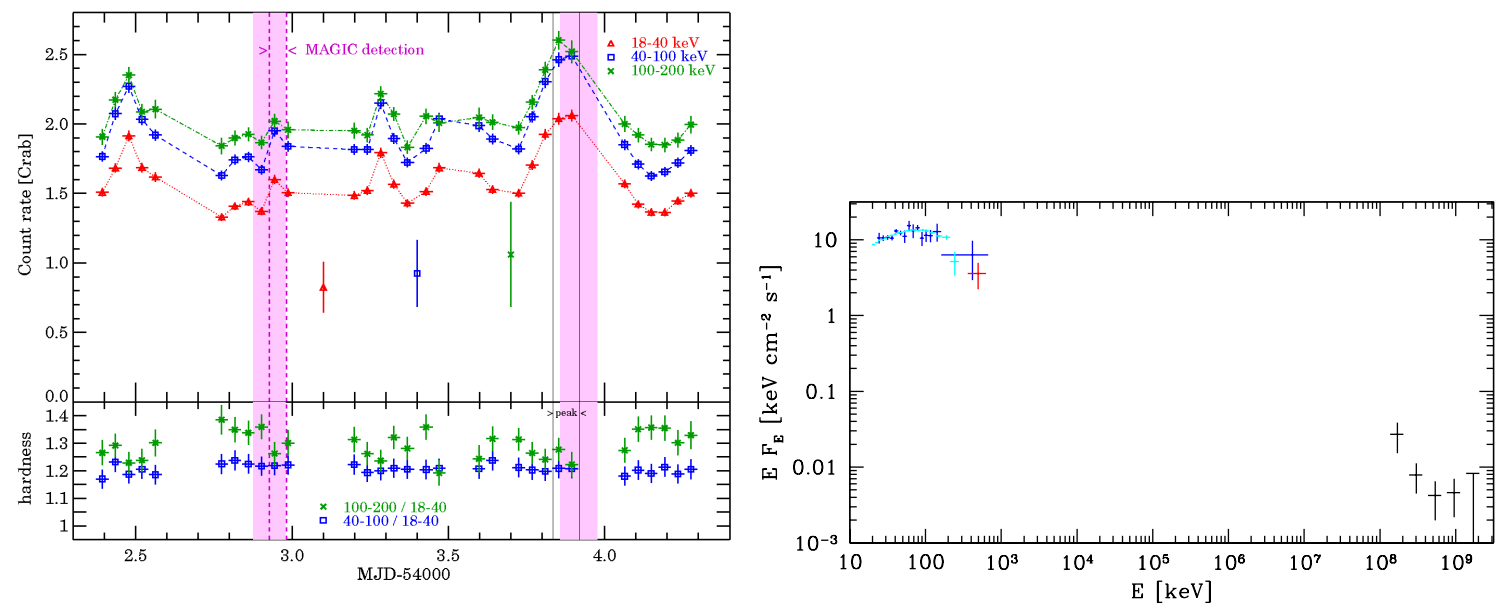

Figure 1: Left: IBIS/ISGRI light curves during rev. 482 (MJD 54002.4-54004.3). The three unconnected symbols (triangle, square and star), around $1 \mathrm{Crab}$, show the historical average IBIS/ISGRI fluxes of Cygnus $\mathrm{X}-1$ in the respective energy bands. The dark vertical stripes indicate the times while the source was observed with the MAGIC telescope. The thick vertical dashed lines mark the start and the end of the MAGIC pointing in which Cyg X-1 was detected at TeV energies. Right: Simultaneous INTEGRAL/MAGIC spectrum of Cygnus X-1: the SPI (blue), ISGRI (cyan) and PICsIT (red) spectra are averaged over the time of the TeV (crosses) detection. See text for details; from Malzac et al. (2008).

The $40-100 \mathrm{keV}$ to $18-40 \mathrm{keV}$ (HR1) and the $100-200 \mathrm{keV}$ to $18-40 \mathrm{keV}$ (HR2) Hardness Ratios (HRs) are shown in the bottom panel of Fig. 1 (left, bottom). Both HRs are remarkably stable: the spectrum remains impressively constant in spite of relatively strong flux fluctuations. The best-fit parameters with all instruments are similar to those obtained using SPI alone, before, during and after the flare: the spectrum during the outburst is consistent with a stable Low/Hard State. Despite variations in the flux by a factor of $\sim 2$ in the the $20-700 \mathrm{keV}$ energy band, the shape of the energy spectrum remained remarkably stable. It is very well represented by an efolded power law with the photon index of $\Gamma \sim 1.4$ and a high energy cut-off at $E_{\mathrm{c}} \sim 130-140 \mathrm{keV}$. The spectrum is also well described by thermal Comptonisation (COMPPS) including a moderate reflection component, with the solid angle of the reflector of $\sim 0.4 \times 2 \pi$. The temperature of the hot Comptonising electrons is $k T_{\mathrm{e}} \sim 70 \mathrm{keV}$ and their Thomson optical depth is $\tau \sim 2.5$. These 
spectral properties are typical of those observed in the low/hard state. This shows that Cygnus X-1 may stay in the low hard state at least up to the flux level of $2 \mathrm{Crab}$, which corresponds to $\sim 2-3$ $\%$ of the Eddington luminosity.
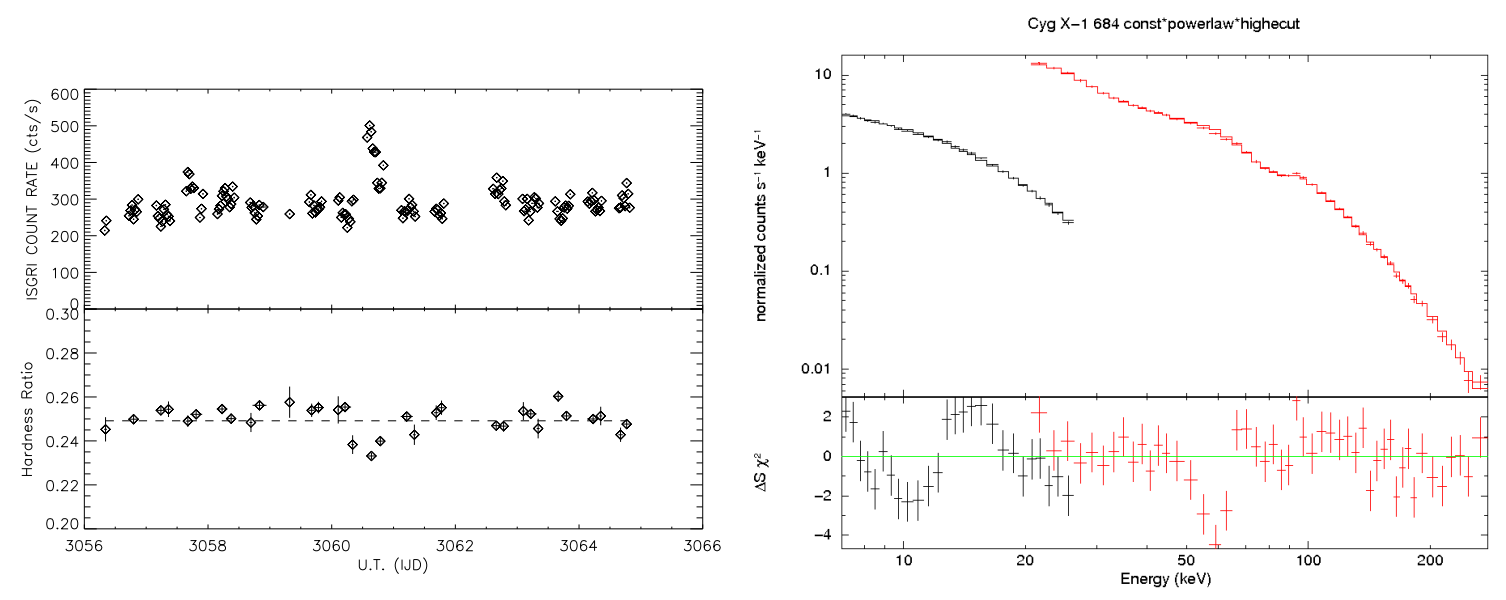

Figure 2: Left: IBIS/ISGRI light curves (20-320 keV) and HR (80-60 keV/20-40 keV) during the 2008 May 18 flare (MJD 54556-54565). Right: 3-day zoom on the corresponding INTEGRAL spectra. From Cadolle Bel et al. (2009a).

Fig. 1 (Right) shows the spectrum of the source at the time of the MAGIC detection, together with the simultaneous $\mathrm{TeV}$ data. Non-thermal high energy components in excess of thermal Comptonisation models have been reported in the hard state of Cygnus X-1 (McConnell et al. 2000, 2002; Cadolle Bel et al. 2006). Such a hard tail is usually attributed to the presence of a small fraction of non-thermal electrons in the hot comptonising medium. In the present data the presence of such an excess is suggested by the shape of the SPI residuals above $300 \mathrm{keV}$, but fitting it with a non-thermal Comptonizing electron distribution did not lead to any sensible improvement of the Chi-squared. This excess does not appear in the PICsIT data. Although this depends on the poorly constrained normalisation factor between SPI and PICsIT, we conclude that, even though it is not excluded, we do not find robust evidence for the presence of a non-thermal excess in this observation. Neronov et al. (2008) reported a more recent flare on May 18th: the source flux reached 2 Crab in the 20-80 $\mathrm{keV}$ range during 8 hours (Fig. 2, left), with a spectrum photon index of $1.55 \pm 0.03$ and cut-off at $161 \pm 13 \mathrm{keV}$ (Fig. 2, right). However, it was substantially weaker (no correspondence to a local flux maximum of the ASM/BAT light curves), and on a shorter time scale than the 2006 event. Further results will be reported in Cadolle Bel et al. (2009a).

\subsection{GRS 1915+105}

We focus on 3 particular observations during which we observe several types of soft X-ray dips and spikes cycles, followed by radio flares. During these observations GRS $1915+105$ is in the so-called $v, \lambda$, and $\beta$ classes of variability (see Fig. 3). The observation of ejections during class $\lambda$ are the first ever reported (Rodriguez et al. 2008a). We then decompose the light curves and corresponding spectra during the dips/spikes (Fig. 4). The fits of each sequence tell us that the disk approaches the $\mathrm{BH}$ monotonically during the cycles, with a flux increasing throughout the whole sequence; the corona flux increases from I to II, decreases from II to III and then slowly recovers 

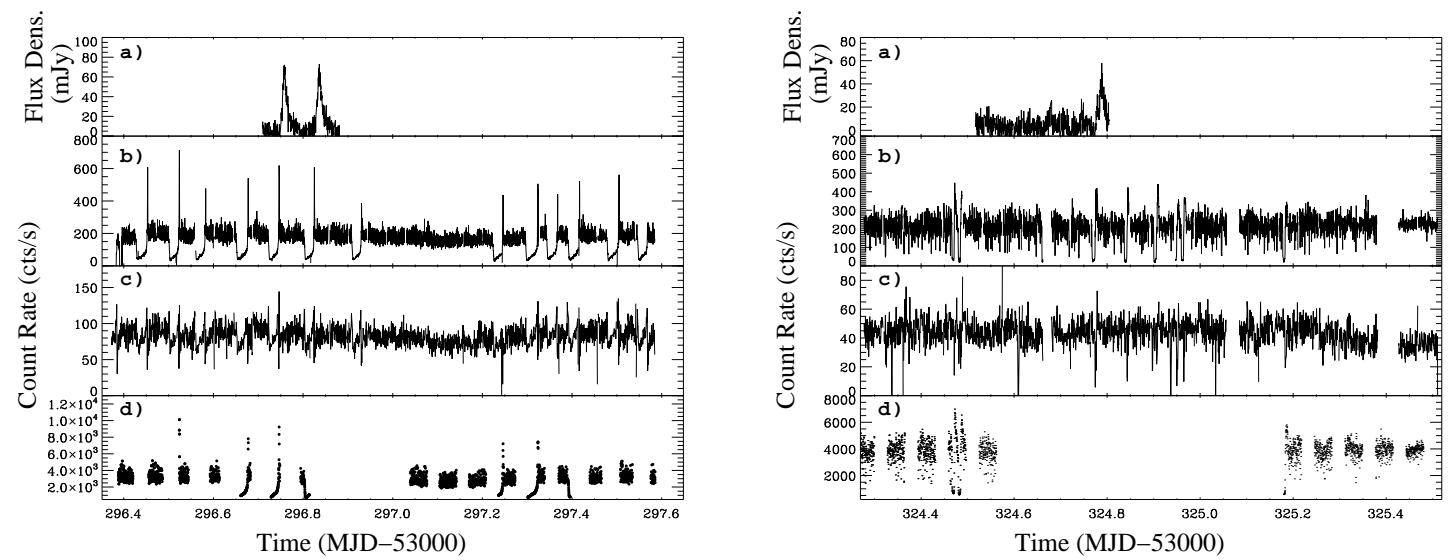

Figure 3: Sequences of dips and spikes in the light curves of GRS 1915+105 (from top to bottom: Ryle, JEM-X, ISGRI and PCA). Left: classes $v$ and $\rho$. Right: classes $\mu, \lambda$ and $\gamma$ (identified with color-color diagrams, not shown here). From Rodriguez et al. (2008a).

from III to IV. We speculate that dip III following precursor spike II indicates the disappearance of the corona, probably ejected and responsible for the observed hard X-rays. Our model independent approach of the high energy data allow us to generalize the fact that a (non-major) discrete ejection always occurs, in GRS $1915+105$, as a response to an X-ray sequence composed of a spectrally hard X-ray dip (more pronounced at soft X-rays) terminated by an X-ray spike marking the disappearance of the hard X-ray emission above $18 \mathrm{keV}$. This model also permits us to identify the trigger of the ejection as this X-ray spike. Similar spectral evolutions are seen for other classes with cycles.
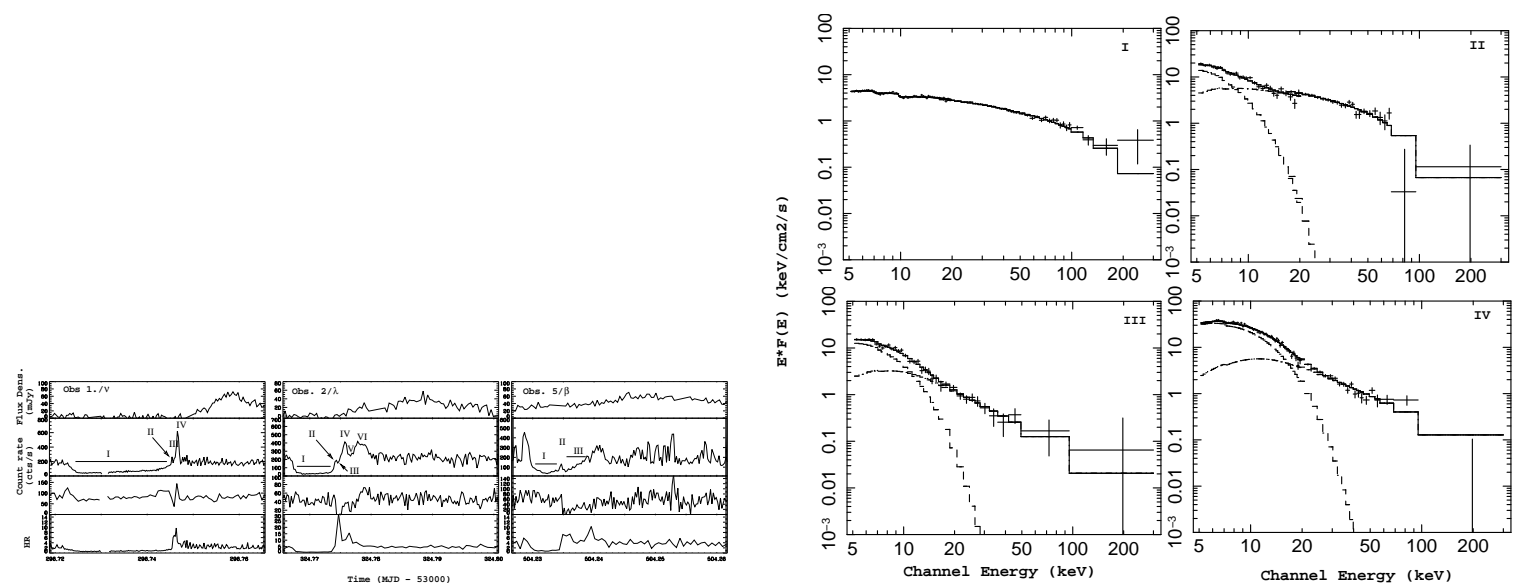

Figure 4: Left: zoom on one cycle from each of the 3 observations with cycles. From top to bottom, the panels respectively represent the Ryle at $15 \mathrm{GHz}$, the JEM-X (3-13 keV), the ISGRI (18-50 keV) lightcurves and the 3-13 keV/18-50 keV HR. The different intervals from which spectra were extracted are indicated with number on the JEM-X light curve. Right: joint JEM-X and ISGRI spectra (disk+Comptonization fit) from these intervals on Obs 1/class $v$. From Rodriguez et al. (2008b).

In addition, a possible correlation between the amplitude of the radio flare and the duration of the X-ray dip is found in our data (Fig. 5, left). In this case, the X-ray dips prior to ejections 
could be seen as the time during which the source accumulates energy and material that is ejected later. Thus, the longer the dip, the more energy and/or matter available for the ejection, and hence the larger the amplitude of the radio flare. Besides, during a period of steady "hard" X-ray state (the so-called $\chi$ class) in which, as expected, a strong Low Frequency (LF) QPO was detected, we observe a steady radio flux. This is interpreted as the signature of the presence of a compact jet. The resultant "QPO spectrum" (Fig. 5, right) is not well fitted by a power law. The energetic dependencies of the QPO amplitude and the relative contribution of the Comptonized component are clearly different. This indicates that the origin of the LFQPO is probably not in the hard tail/the jet and rules out global oscillations of the corona (see Rodriguez et al. 2008a,b for more details).
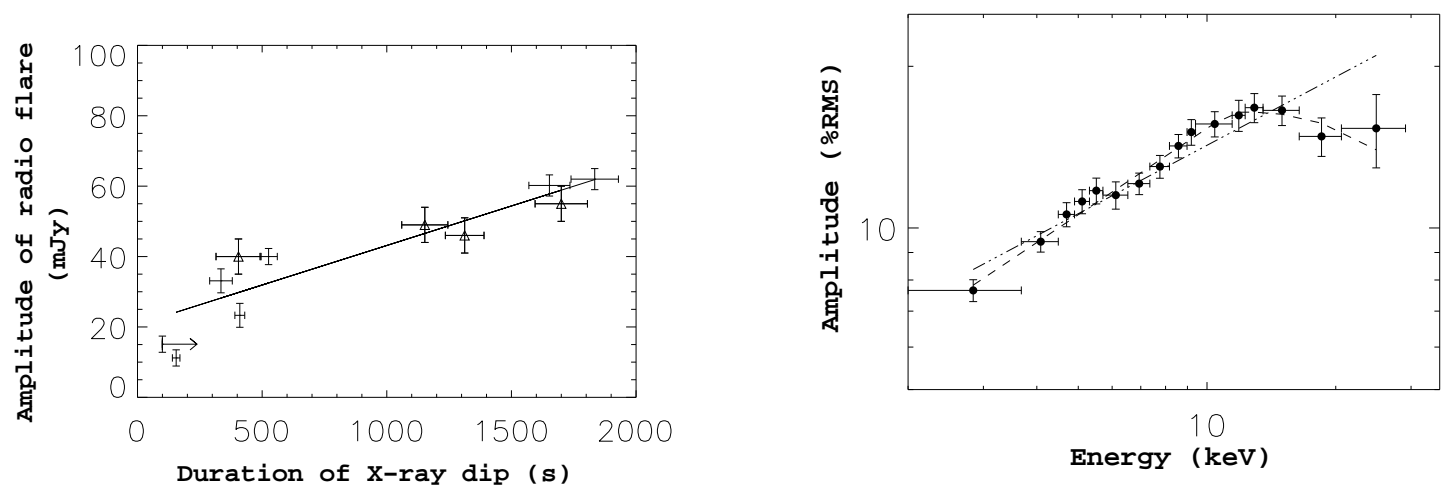

Figure 5: Left: Evolution of the amplitude of the radio flares vs. the duration of the preceding X-ray dip. The points are from this study and the triangles come from Pooley \& Fender (1997) and Klein-Wolt et al. (2002). The line represents the linear function that best fits the data. Right: Spectrum of the LFQPO from the unique RXTE observation that was simultaneous to INTEGRAL ( $\chi$ class) and the Ryle. The best model obtained from fits with power law and power law with an exponential cut-off are respectively over-plotted as a dash-dotted and a dashed line. A better fit is obtained with the power law with cut-off. From Rodriguez et al. (2008a,b).

\section{Discussion}

It is the first time that Cygnus X-1, a persistent high-mass black-hole binary, is observed twice at a few percent of the Eddington luminosity with a stable Low/Hard State spectrum over a period of a few days. Such a bright hard state has so far been observed only during the rising phase of transient low-mass black-hole binaries. The INTEGRAL flare is exceptional in terms of duration. Were it above the soft-state level flux, it would, most likely, have triggered a state transtion to the soft state during the 2-day time scale. The TeV detection coincides with the peak of a small Xray flare just after a very fast rise in hard X-ray flux. In contrast, the source remained undetected by MAGIC at the peak of a larger X-ray flare occurring one day later and corresponding to the maximum of the X-ray luminosity of the whole outburst. We do not find any obvious correlation between the $\mathrm{X}$-ray and $\mathrm{TeV}$ emission, which could in principle be related to non-thermal coronal electrons (for more details on this emission processes and modeling, see Malzac et al. 2008 and Zdziarski et al. 2008). Alternatively, the $\mathrm{TeV}$ emission could arise in shocks located in the region 
where the outflow originating close to the black hole interacts with the wind of the star. Such an outflow is indeed observed in Cygnus X-1 in the form of the compact radio jet. Other forms of outflows such as an accretion disc wind could also be responsible for this emission. This outflow colliding with the star wind may power strong shocks accelerating the particles responsible for the $\mathrm{TeV}$ emission. In this scenario an increase of the wind strength would increase the mass accretion rate onto the black hole, leading to the enhanced hard X-ray emission. Simultaneously the stronger wind interacts more efficiently with the (possibly stronger) outflow, making the $\mathrm{TeV}$ emission detectable. Still, pair absorption in the photon field of the companion (e.g., Dubus 2006) remains to be a major issue.

The fact that the results on GRS 1915 do not rely on any spectral modelling enhances their robustness. We show that bubble ejections always follow a sequence of a hard dip terminated by a sudden spike at X-ray energies (referred to as a cycle). Comparing many classes and other sources (Rodriguez et al. 2003), this seems to be a very generic phenomenon in microquasars. Spectral and timing analysis of the unique observation during which GRS 1915+105 is found in a steady hard-intermediate state showed a strong and rather steady jet is detected in radio. The QPO does not have its origin in the jet, and may also rule out models based on oscillations of a Compton corona. Globally the behavior of the LFQPO is more compatible with models of disk instabilities. The strong LFQPO detected is compatible with the prediction of the AEI and the magnetic flood proposed to explain the behavior of GRS $1915+105$ during class $\beta$ (Tagger et al. 2004). The AEI may also well explain the regularity observed in the regular succession of classes of the source over 11 years that we have found (Cadolle Bel et al. 2009b, in prep.).

Only by improving the coverage and quality of such multiwavelength campaigns like the ones presented in this work can one understand the connections and origin of the hard X-ray emitters to those of the radio jets. The correlations between X-rays and radio can then be discussed and detailed (e.g., Cadolle Bel et al. 2007) for many other sources so as to get a complete picture of the physical mechanisms responsible for accretion and ejection in microquasars.

\section{References}

[1] Albert, J., and the MAGIC collaboration, 2007, ApJ, 665, L51

[2] Belloni, T., Mendez, M., King, A. R., et al. 1997, ApJ, 488, 109

[3] Belloni, T., Klein-Wolt, M., Mendez, et al. 2000, A\&A, 355, 271 et al. 2000

[4] Bowyer, S., Byram, E. T., Chubb, T. A., et al. 1965, Sci, 147, 394

[5] Cadolle Bel, M., Goldwurm, A., Rodriguez, J., et al. 2006, A\&A, 446, 591

[6] Cadolle Bel, M., Ribó, M., Rodriguez, J., et al. 2007, ApJ, 659, 549

[7] Dubus, G. 2006, A\&A, 451, 9

[8] Gies, D. R., \& Bolton, C. T., 1986, ApJ, 304, 371

[9] Herrero A., Kudritzki, R. P., Gabler, R., et al. 1995, A\&A, 297, 556

[10] Homan, J. \& Belloni, T. 2005, Ap\&SS, 300, 107

[11] Klein-Wolt, M., Fender, R. P., Pooley, G. G., et al. 2002, MNRAS, 331, 745 
[12] Malzac, J., Petrucci , P. O., Jourdain, E., et al. 2006, A\&A, 448, 1125

[13] Malzac et al. 2008, accepted by A\&A, astro-ph 0805.4391

[14] McConnell, M. L., Ryan, J. M., Collmar, W., et al. 2000, ApJ, 543, 928

[15] McConnell, M. L., Zdziarski, A. A., Bennett, K., et al. 2002, ApJ, 572, 984

[16] Morgan, E. H., Remillard, R. A. \& Greiner, J. 1997, ApJ, 482, 993

[17] Neronov et al. 2008, Cadolle Bel, M., Shaw, S., et al. 2008, ATel 1533, 1 (errata in ATel 1536, 1)

[18] Pooley, G. G, \& Fender, R. P. 1997, MNRAS, 292, 925

[19] Rodriguez, J., Durouchoux, P., Tagger, M., et al. 2002, A\&A, 386, 271

[20] Rodriguez, J., Corbel, S. \& Tomsick, J.A. 2003, ApJ, 595, 1032

[21] Rodriguez, J., Hannikainen, D., Shaw, S. E., et al. 2008a, ApJ, 675, 1436

[22] Rodriguez, J., Shaw, S. E., Hannikainen, D., et al. 2008b, ApJ, 675, 1449

[23] Tagger, M., Varnière, P., Rodriguez, J. \& Pellat, R. 2004, ApJ, 607, 410

[24] Vadawale, S. V., Rao, A. R., Naik, S., et al. 2003, ApJ, 597, 1023

[25] Zdziarski, A. A., Poutanen, J., Paciesas, W. S., et al. 2002, ApJ, 578, 357

[26] Zdziarski et al. 2008, submitted to MNRAS, astro-ph 0809.3255

[27] Ziólkowski, J. 2005, MNRAS, 358, 851 